\begin{tabular}{|c|c|c|}
\hline 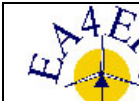 & $\begin{array}{c}\text { European Association for the } \\
\text { Development of Renewable Energies, Environment } \\
\text { and Power Quality (EA4EPQ) }\end{array}$ & $\begin{array}{l}\text { International Conference on Renewable Energies and Power Quality } \\
\text { (ICREPQ'12) } \\
\text { Santiago de Compostela (Spain), 28th to 30th March, } 2012\end{array}$ \\
\hline
\end{tabular}

\title{
Analysis of Newton-Like Extremum Seeking Control in Photovoltaic Panels
}

\author{
H. Zazo, R. Leyva and E. del Castillo \\ Department of Electronic, Electrical and Automatic Control Engineering \\ University Rovira i Virgili \\ Av. Paisos Catalans, 43007 Tarragona (Spain) \\ Phone/Fax number:+0034 977558520/9605, e-mail: ramon.leyva@urv.cat
}

\begin{abstract}
The paper adapts the NL-ESC technique to extract the maximum power from photovoltaic panels. This technique uses the gradient and Hessian of the panel characteristic in order to approximate the operation point to the optimum. The paper describes in detail the gradient and Hessian estimations carried out by means of sinusoidal dithering signals. Furthermore, we compare the proposed technique with the common ESC that only uses the gradient. The comparison is done by means of PSIM simulations and it shows the different transient behaviors and the faster response of the NL-ESC solution.
\end{abstract}

Keywords. Photovoltaics, Maximum Power Point Tracking, Extremum Seeking Control, Newton Method.

\section{Introduction}

Maximum Power Point Tracking (MPPT) algorithms allow photovoltaic systems to operate efficiently. For such a purpose, many authors have proposed different MPPT methods [1]. These methods have deserved attention from the Power Electronics field but also from the Automatic Control domain. In the domain of Control Theory, the technique that drives systems to their optimal operation point is named Extremum Seeking Control (ESC). Such techniques appear during the fifties [2] and most of them use the block schema shows in figure 1 .

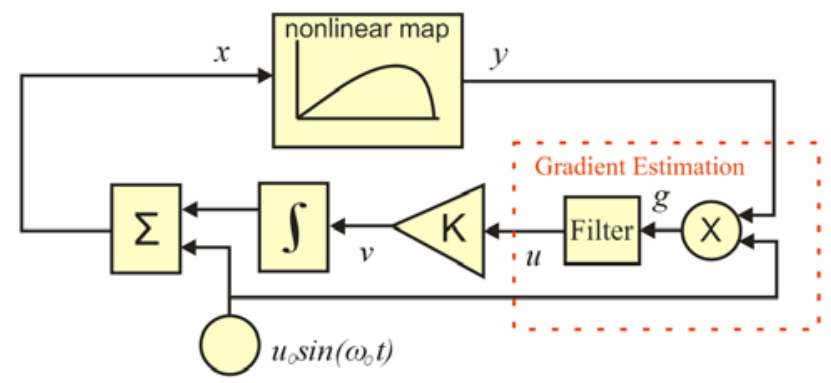

Fig. 1. ESC schema
Several authors have described MPPT circuits based on ESC for photovoltaic application [3-7]. Common ESC technique allows systems to approximate with a speed proportional to the characteristic curvature; they work correctly under the constraint that such characteristic is concave [2]. Consequently, certain control signals depend on the characteristic curvature. In photovoltaic panels, the curvature of voltage-power characteristic is large when the point is at the right-side of the maximum. This fact may cause some implementation drawbacks as the saturation of certain signals and undesired transient behaviours.

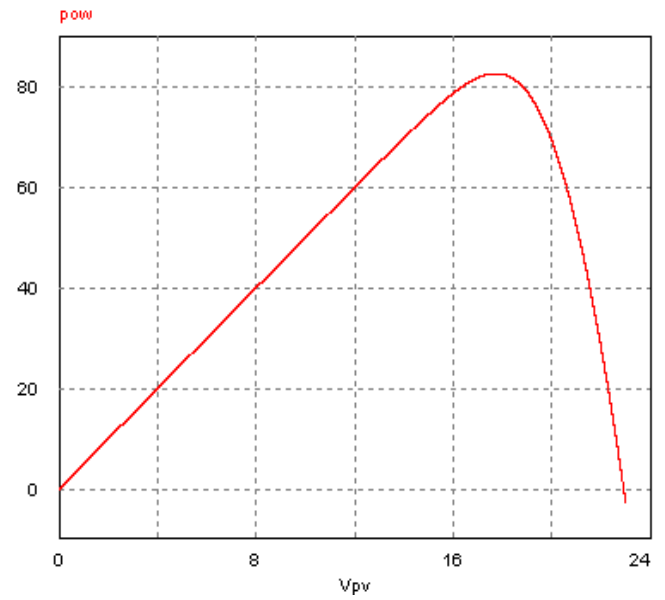

Fig. 2. Voltage-Power Characteristic in Photovoltaic Panel BP-585

Recently, Moase et alt. [8] proposed a new control algorithm called Newton-Like Extremum Seeking Control (NL-ESC) in order to that the approximation speed do not depend on the characteristic curvature, thus improving the aforementioned drawbacks. This algorithm estimates the characteristic Hessian with four dithering signals perfectly synchronized (namely, $\sin (\omega t), \cos (\omega t), \sin (2 \omega t)$ and $\cos (2 \omega t))$. Furthermore, Moase describes a dynamic 
adaptation mechanism for the amplitude of the dithering signals.

Our paper adapts the NL-ESC to extract the maximum energy from a photovoltaic panel, according to figure 3; and in addition, our proposed method differs from Moase's one since it do not need a synchronization of the dithering signals.

The paper is organized as follows; in section 2, we revisit the basis of NL-ESC; specifically, we describe the control schema and the operating principles. In section 3, we compare the NL-ESC and the common ESC in MPPT circuits for photovoltaic generation. Finally, we summarize the main conclusion in section 4 .

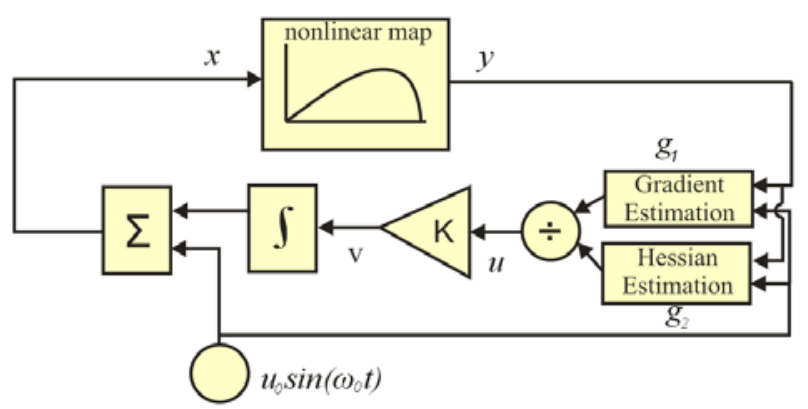

Fig. 3. NL-ESC schema

\section{MPPT based on NL-ESC Method}

NL-ESC requires from a Hessian estimation of voltagepower characteristic. In this section, we describe, first, the Hessian estimation and, then, the rest of block diagram of the proposed MPPT.

\section{A. Estimation of the PV Panel Hessian}

Given a non-linear panel characteristic,

$$
y=f(x)
$$

where $y$ is the output power and $x$ represents the generator input. Considering that the input $x$ consist of a slow signal $u$ and a sinusoidal dithering of small amplitude $u_{o}$ and angular frequency $\omega_{0}$; that is,

$$
x=u+u_{0} \sin \left(\omega_{0} t\right)
$$

then the output power expression corresponds to,

$$
y=f\left(u+u_{0} \sin \left(\omega_{0} t\right)\right)
$$

The power expression can be approximated by the first terms of Taylor series,

$y \approx f(u)+\frac{d f(u)}{d u} u_{0} \sin \left(\omega_{0} t\right)+\frac{1}{2} \frac{d^{2} f(u)}{d u^{2}} u_{0}^{2} \sin ^{2}\left(\omega_{0} t\right)$

$$
\begin{aligned}
y \approx & f(u)+\frac{d f(u)}{d u} u_{0} \sin \left(\omega_{0} t\right)+\frac{1}{4} \frac{d^{2} f(u)}{d u^{2}} u_{0}{ }^{2} \\
& -\frac{1}{4} \frac{d^{2} f(u)}{d u^{2}} u_{0}{ }^{2} \cos \left(2 \omega_{0} t\right)
\end{aligned}
$$

It can be observed that the panel output is made up of a low frequency component, a first harmonic component at $\omega_{0}$ and a second harmonic component at $2 \omega_{0}$. The information about the curvature or Hessian of the panel characteristic is associated to the second harmonic. Then, in order to extract that information, we apply a high-pass filter $G(s)$ to output power $y$; that is,

$$
y_{f} \approx \frac{d f(u)}{d u} u_{0} \sin \left(\omega_{0} t\right)-\frac{1}{4} \frac{d^{2} f(u)}{d u^{2}} u_{0}^{2} \cos \left(2 \omega_{0} t\right)
$$

and multiplying the filtered output $y_{f}$ by $u_{0}{ }^{2} \cos ^{2}\left(\omega_{0} t\right)$, we obtain

$$
\begin{aligned}
& g_{2} \approx \frac{1}{2} \frac{d f(u)}{d u} u_{0}^{3} \sin \left(\omega_{0} t\right)+\frac{1}{4} \frac{d f(u)}{d u} u_{0}^{3} \sin \left(3 \omega_{0} t\right) \\
& -\frac{1}{4} \frac{d f(u)}{d u} u_{0}^{3} \sin \left(\omega_{0} t\right)-\frac{1}{8} \frac{d f^{2}(u)}{d u^{2}} u_{0}^{4} \cos \left(2 \omega_{0} t\right) \\
& -\frac{1}{16} \frac{d f^{2}(u)}{d u^{2}} u_{0}{ }^{4}-\frac{1}{16} \frac{d f^{2}(u)}{d u^{2}} u_{0}^{4} \cos \left(4 \omega_{0} t\right)
\end{aligned}
$$

Where it can be observed that, now, the Hessian information is associated to continuous component. Consequently, we apply a low-pass filter, $H(s)$, and obtain the estimation of the Hessian

$$
p \approx-\frac{1}{16} \frac{d f^{2}(u)}{d u^{2}} u_{0}^{4}
$$

In order to obtain the signal $p$, which is proportional to the Hessian, we need the multiplying term $u_{0}^{2} \cos ^{2}\left(\omega_{0} t\right)$. This term can be implemented from the dithering signal $u_{0} \sin (\omega t)$ by means of a dc generator plus a fourquadrant analog multiplier, according to,

$$
u_{0}^{2} \cos ^{2}(\omega t)=u_{0}^{2}-u_{0}^{2} \sin ^{2}(\omega t)
$$

Figure 4 shows the Hessian estimation schema that is used to implement the MPPT based on NL-ESC method. It can be observe, in the figure, the high-pass filter, low-pass filter, multiplier element and the implementation of the term $u_{0}^{2} \cos ^{2}\left(\omega_{0} t\right)$.

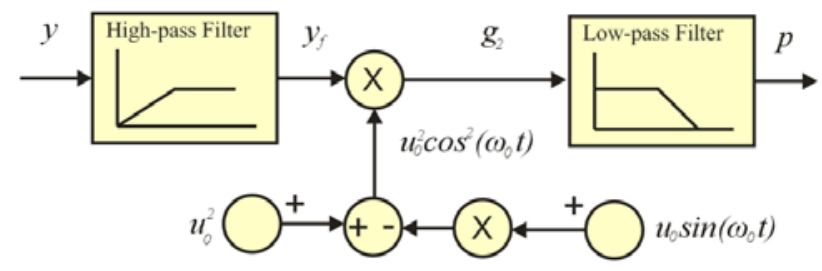

Fig. 4. Hessian estimation schema

That, in turn, it can be rewritten as, 
The low-pass filter in the Hessian estimator correspond to,

$$
H(s)=\frac{H_{0}}{s^{2}+2 \xi_{H} \omega_{0 H} s+\omega_{0 H}^{2}}
$$

and the high-pass filter correspond to,

$$
G(s)=\frac{G_{0} s^{2}}{s^{2}+2 \xi_{G} \omega_{0 G} s+\omega_{0 G}^{2}}
$$

these parameters are instantiated in table II of section 3 .

\section{B. NL-ESC method for MPPT in Photovoltaic domain}

The proposed MPPT based on NL-ESC method operates according to schema of Figure 3, where the input signal $x$ corresponds to the converter duty-cycle. Given that the nonlinear map block represents the PV generator, which consists of a dc-dc converter and PV panel, and the converter and panel transients are much faster than those of the MPPT loop, the PV generator can be modelled by a static nonlinear map. The PV generator operating point depends on the input signal $x$; this signal is the output of an integrator block plus a sinusoidal dithering signal of small amplitude. The input signal $x$ must increase if the PV generator operates at the left of the maximum and must decrease it $x$ is greater than the optimal point $x^{*}$.

We multiply the PV generator output $y$ by the sinusoidal dithering signal and filter it to estimate the gradient; as it can be observer in Fig. 1.

The integrator input $v$ should be proportional to the gradient of the PV generator characteristics in MPPT circuits based on ESC, and proportional to the gradient divided by the Hessian in MPPT circuits based on NLESC.

Figure 3 shows the functional blocks involved in MPPT circuits based on NL-ESC. It can be observed that MPPT circuits using NL-ESC are slightly more complex than MPPT circuits based on ESC.

As it is shown in figure 5, we add some saturation blocks after Hessian estimation sub-circuits, to prevent a very low value of the divisor. Figure 5 depicts a PSIM schematic showing a detail of gradient and Hessian implementation, also it can be observed the placement of the saturation block with bounds $V_{\text {sat }}^{\text {low }}$ and $V_{\text {sat }}^{\text {high }}$.

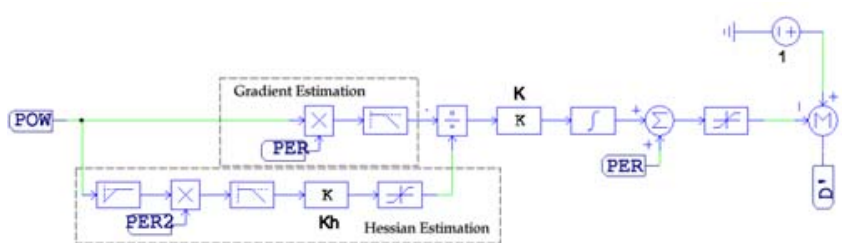

Fig. 5. PSIM Schematic of MPPT circuit based on NL-ESC

The Hessian value is near to zero when the operating point $x$ is at the left of the optimum; as can be appreciated in figure 6 that depicts the gradient and Hessian of the characteristic power versus input voltage in an $85 \mathrm{w}$ PV panel.

Table I: Parameters of the MPPT based on ESC

\begin{tabular}{|c|c|c|c|c|}
\hline \multicolumn{2}{|c|}{$u_{0}$} & $0,01 \mathrm{~V}$ & $\omega_{0}$ & $50 \mathrm{~Hz}$ \\
\hline \multirow{3}{*}{$H(s)$} & $\mathrm{H}_{0}$ & 1 & \multirow{3}{*}{$K$} & \multirow{2}{*}{150} \\
\cline { 2 - 3 } & $\xi_{\mathrm{H}}$ & 1 & & \\
\cline { 2 - 3 } & $\omega_{0 \mathrm{H}}$ & $10 \pi \mathrm{rad} / \mathrm{s}$ & & \\
\hline
\end{tabular}

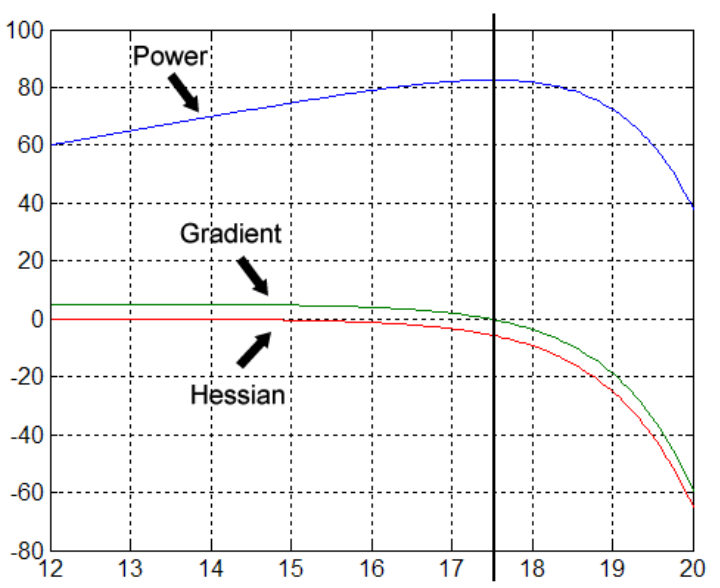

Fig. 6. Voltage-power characteristic of a panel BP585F

We must remark than when MPPT circuit based on NLESC operates at the left of the optimum, it behaves as a MPPT based on ESC since the Hessian term is saturated by $V_{\text {sat }}^{\text {low }}$ and variation of the PV depends only on the gradient. Moreover, the approximation to the maximum depends on the curvature of the characteristic [9]; depends on the Hessian.

In the MPPT based on NL-ESC the approximation to the maximum do not depends on the curvature, since the increasing term $v$ is divided by the Hessian and avoid large values of the increasing term that may cause instabilities in the MPPT circuit that is nonlinear.

\section{Comparison of ESC and NL-ESC MPPT by means of PSIM simulation}

We compare the transient behaviour of both MPPTs for a PV panel BP585F. The nominal peak power is $85 \mathrm{~W}$ and the nominal input voltage is $18.7 \mathrm{~V}$. Table I shows the MPPT parameters of MPPT based on ESC; that is, the dithering amplitude $u_{0}$ and frequency $\omega_{0}$, the filter parameters for $H(s)$ and $G(s)$, and gain constant $K$. Table II shows the parameters of the MPPT based on NL-ESC.

\begin{tabular}{|c|c|c|c|c|c|}
\hline \multicolumn{2}{|c|}{$u_{0}$} & $0,01 \mathrm{~V}$ & \multicolumn{2}{|c|}{$\omega_{0}$} & $50 \mathrm{~Hz}$ \\
\hline \multirow{3}{*}{$H(s)$} & $\mathrm{H}_{0}$ & 1 & \multirow{3}{*}{$G(s)$} & $\mathrm{G}_{0}$ & 1 \\
\hline & $\xi_{\mathrm{H}}$ & 1 & & $\xi_{\mathrm{G}}$ & 1 \\
\hline & $\omega_{0 \mathrm{H}}$ & $10 \pi \mathrm{rad} / \mathrm{s}$ & & $\omega_{0 \mathrm{G}}$ & $60 \pi \mathrm{rad} / \mathrm{s}$ \\
\hline \multirow{2}{*}{\multicolumn{2}{|c|}{$K_{h}$}} & \multirow{2}{*}{3000} & \multirow{2}{*}{$\begin{array}{l}\text { Sat. } \\
\text { bounds }\end{array}$} & $V_{\text {sat }}^{\text {low }}$ & 0,001 \\
\hline & & & & $V_{\text {sat }}^{\text {high }}$ & 0,2 \\
\hline \multicolumn{2}{|c|}{$K$} & 0,15 & & & \\
\hline
\end{tabular}

Table II: Parameters of the MPPT based on NL-ESC. 

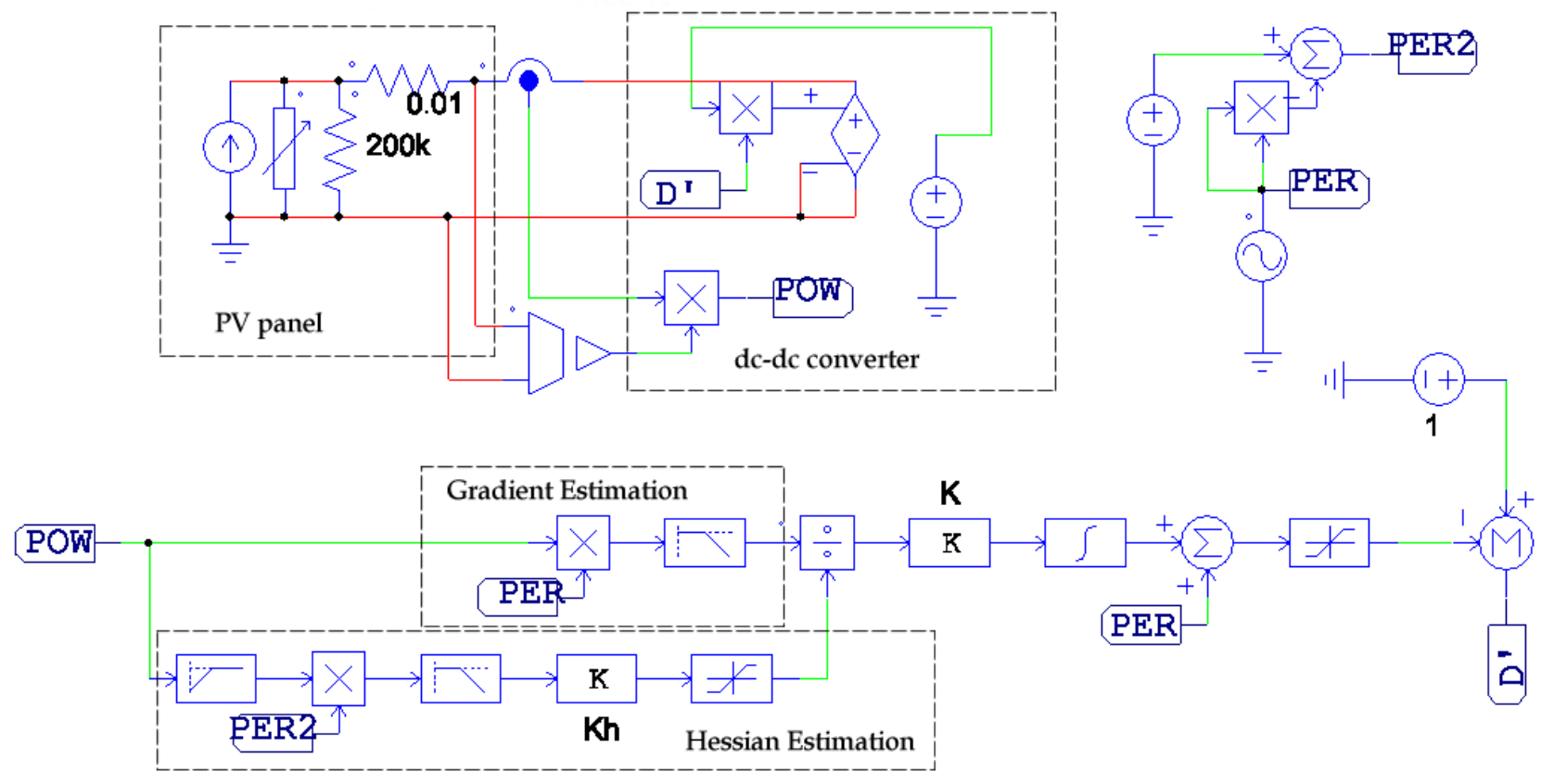

Fig.7. PSIM schematic of the solar system in NL-ESC case

We choose a loop gain constant $K$ equal to 150 in ESC case. The loop gain constant that corresponds to $K_{N L}=K / K_{h}$ is inside the interval $[0.75,150]$ in the NL-ESC case.

The PSIM schematic that consists of a PV panel, a boost dc-dc converter, and the blocks of the MPPT based on NL-ESC; it is shown in figure 7.

Blocks corresponding to the MPPT based on ESC are shown in figure 8 ,

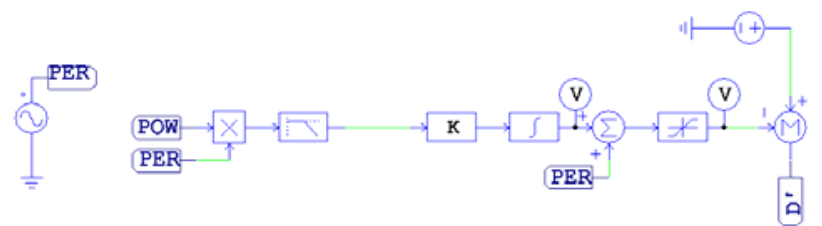

Fig. 8. PSIM schematic of MPPT in ESC case

The following subsections show the transient when the panel input voltage is at the left side of the optimal voltage and when it is at the right side. We have taken voltages far away of the optimum, corresponding to a boost-converter duty cycle $D_{i}=1$ (which is equivalent $0 \mathrm{~V}$ in the panel) and $D_{i}=0,15$ (equivalent $20,4 \mathrm{~V}$ in the panel).

\section{A. Transient waveform starting at $D_{i}=1$}

The Hessian value is near to zero when the panel voltage is low, as it is depicted in figure 6, then the Hessian term is saturated to $V_{\text {sat }}^{\text {low }}$. This is the reason why both MPPTs waveforms are very similar. Waveforms corresponding to the duty-cycle transient and panel power transient are shown in figures 9 and 10, respectively. Nonetheless, a little difference can be appreciated when the voltage is near to the optimum and Hessian term is bigger than the saturation low limit $V_{\text {sat }}^{\text {low }}$.

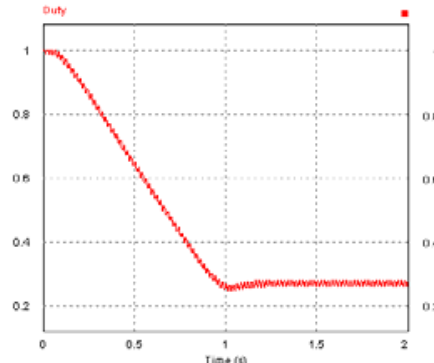

a)

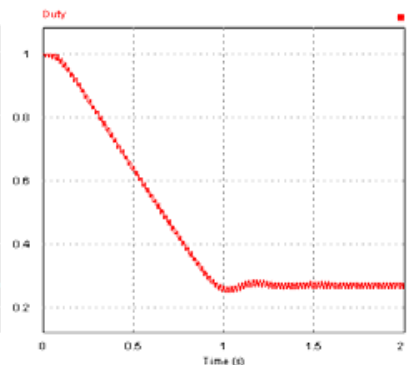

b)
Fig. 9. a) Transient of duty-cycle starting at $D_{i}=1$ in NL-ESC. b) transient of duty-cycle starting at $D_{i}=1$ in ESC.

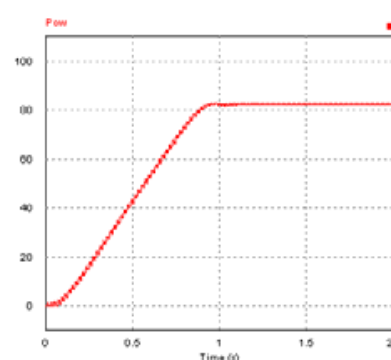

a)

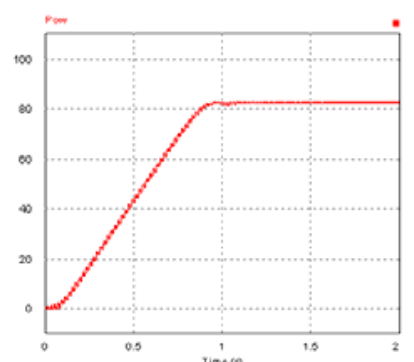

b)
Fig. 10. a) Transient of output power from $D_{i}=1$ in NL-ESC. b) Transient of output power from $D_{i}=1$ in ESC. 


\section{B. Transient waveform starting at $D_{i}=0,15$}

A low starting value of the duty-cycle in the boost converter implies a high voltage in the panel port. This means, according to figure 6 , that the Hessian is big in absolute value terms and the Hessian term is not saturated in the NL-ESC case. The loop gain constant $K_{N L}$ will move inside the interval $[0.75,150]$ then the transient behavior will be different to that of the ESC where the loop gain constant $K$ is 150 . Figures 11 and 12 show the transient waveform of duty-cycle and output power respectively for both MPPT approaches.

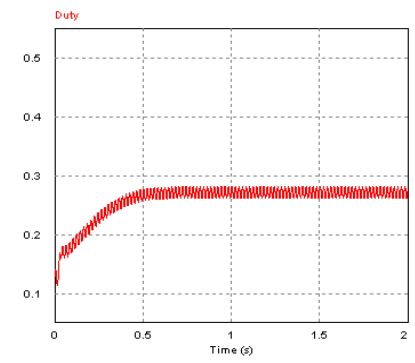

a)

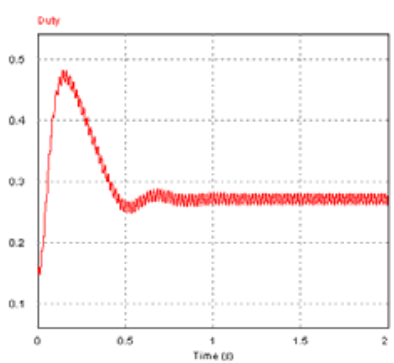

b)
Fig. 11. a) Transient of duty-cycle from $D_{i}=0,15$ in NL-ESC. b) transient of duty-cycle from $D_{i}=0,15$ in ESC.

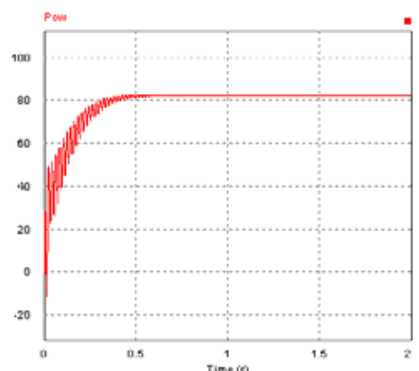

a)

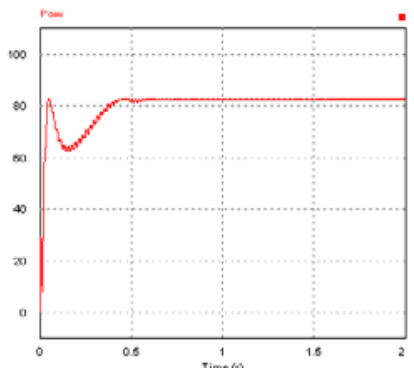

b)
Fig. 12. a) Transient of panel power from $D_{i}=0,15$ in NL-ESC. b) transient of panel power from $D_{i}=0,15$ in ESC.

We can choose a very good value of loop-gain constant $K$ of the MPPT based on ESC for a given starting point and some given weather conditions. Nevertheless, when the starting point or weather conditions change, this value may be too big and therefore the system will become instable or too small and then the transient will be very slow. On the contrary, the equivalent loop-gain constant $K_{N L}$ do not depend on starting point or weather condition and therefore we overcome these drawbacks. We illustrate these different behaviors in the following subsection.

\section{Response of MPPT approaches in front of weather conditions change}

We compare the response of both MPPTs in front of changes of temperature and irradiation. A fast temperature change will involve a fast change of the optimal panel voltage and the MPPT circuits should react in a fast and reliable manner. Figures 13 and 14 depicts MPPT waveforms in front an abrupt change of temperature, both MPPTs reach the optimal point nevertheless the transient is different.

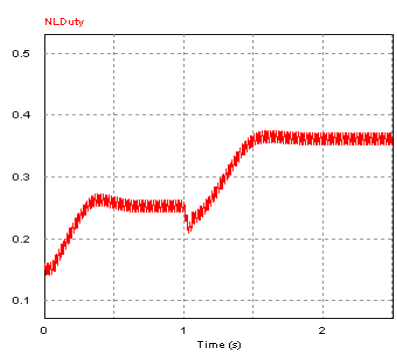

a)

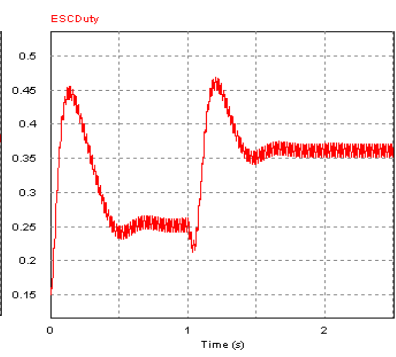

b)
Fig. 13. Transient of the duty-cycle in front an abrupt change of temperature. a) in NL-ESC case. b) in ESC case.

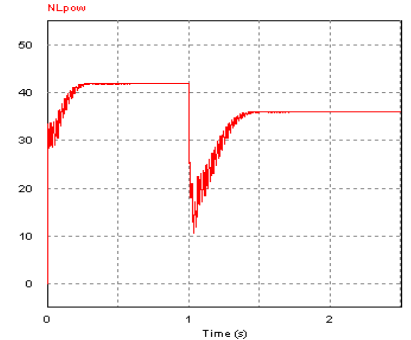

a)

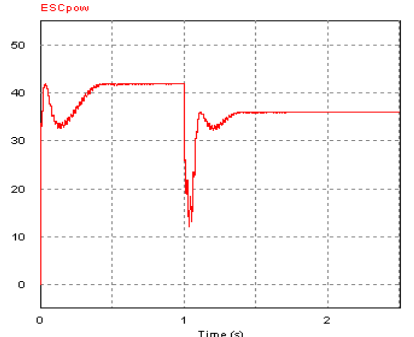

b)
Fig. 14. Transient of panel power in front an abrupt change of temperature. a) in NL-ESC case. b) in ESC case.

Irradiance changes are very often in terrestrial PV systems; this is the reason why MPPT circuits should react in a fast and reliable manner. When irradiance changes the voltage optimum shift slightly, however the power output change in a great extent and then MPPT signal changes abruptly also when the MPPT operating point is near to the optimum. We can appreciate in figures 15 and 16 that both MPPT approach react in a correct way in front an abrupt change of irradiance. We can appreciate that the transient are different.

Nevertheless, an abrupt increase in irradiance may cause an unstable behavior, this is because during the transient the panel voltage exceeds the open-voltage value and the MPPT then is not able to find the gradient of the power since it is zero.

Due to the fact that the equivalent loop-gain constant is adapted in the NL-ESC approach, the NL-ESC waveforms have less overshoot and then this approach is a more stable approach as figures 17 and 18 corroborate.

Figures 15 and 16 show that the optimal panel voltage changes slightly and they also show a stable behavior. On the contrary, figures 17 and 18 depict a small change of optimal panel voltage (or the equivalent duty-cycle) in the NL-ESC approach but also illustrate that the ESC approach becomes unstable. This simulations show that an approach that takes into account the Hessian or curvature of the panel power is more reliable. 


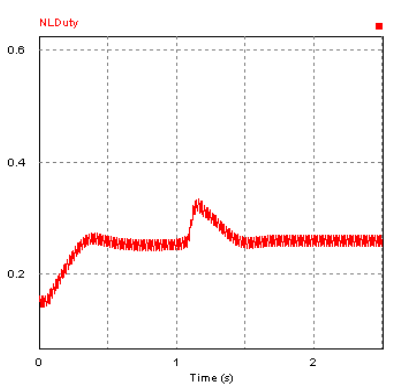

a)

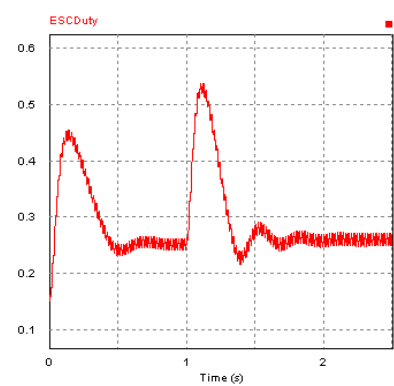

b)
Fig. 15. Transient of duty-cycle in front an abrupt irradiance decrease. a) in NL-ESC case. b) in ESC case.

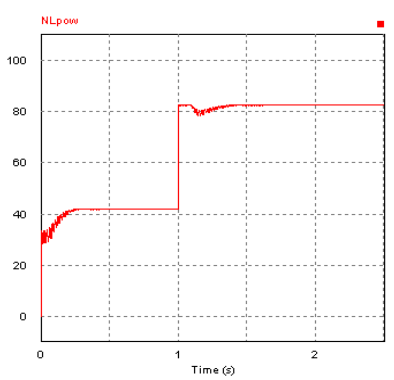

a)

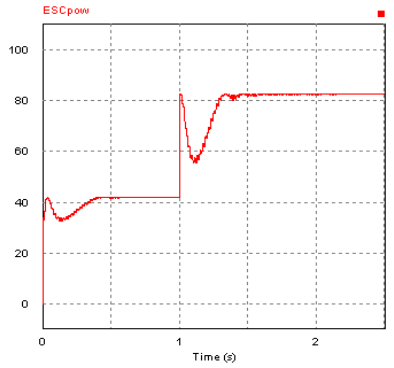

b)
Fig. 16. Transient of panel power in front an abrupt irradiance decrease. a) in NL-ESC case. b) in ESC case.

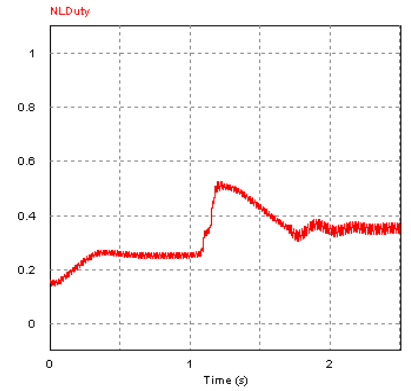

a)

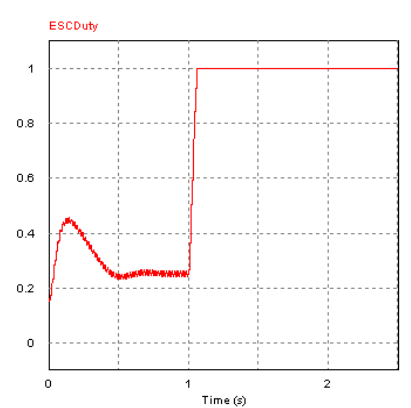

b)
Fig. 17. Transient of duty-cycle in front an abrupt irradiance increase. a) in NL-ESC case. b) in ESC case.

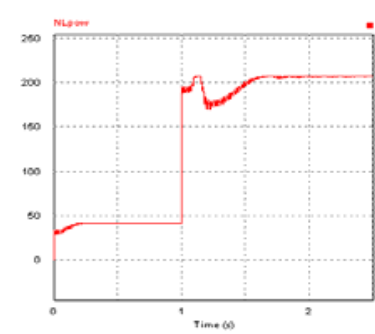

a)

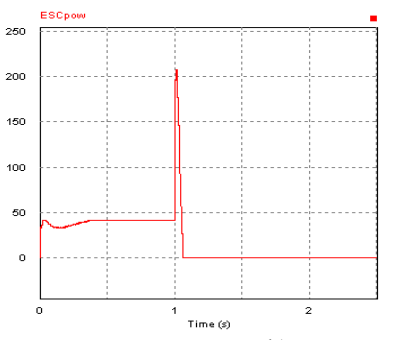

b)
Fig. 18. Transient of panel power in front an abrupt irradiance increase. a) in NL-ESC case. b) in ESC case.
Control. The MPPT circuits based on both approaches have been simulated with PSIM. We corroborate the different transient behavior. Simulation waveforms verify that the MPPT based on Newton-like Extremum Seeking Control is more stable. An experimental implementation of the proposed approach is in progress.

\section{Acknowledgment}

This work was partially supported by the Spanish Ministerio de Educación y Ciencia under grant DPI201016481.

\section{References}

[1] Esram, T.; Chapman, P.L; , "Comparasion of Photovoltaic Array Maximum Power Point Tracking Techniques", Energy Conversion, IEEE Transactions on, vol.22, pp.439, Jun. 2007

[2] Ariyur, K. B., and Krstic, M., "Slope Seeking and Application to Compressor Instability Control", Decision and Control, 2002, Proceedings of the 41st IEEE Conference on, vol. 4.

[3] Leyva, R.; Alonso, C.; Queinnec, I.; Cid-Pastor, A.; Lagrange, D.; Martinez-Salamero, L.; , "MPPT of photovoltaic systems using extremum - seeking control," Aerospace and Electronic Systems, IEEE Transactions on, vol.42, no.1, pp. 249- 258, Jan. 2006

[4] Olalla, C.; Arteaga, M.I.; Leyva, R.; El Aroudi, A., "Analysis and Comparison of Extremum Seeking Control Techniques", Industrial Electronics, 2007. ISIE 2007. IEEE International Symposium on, Jun. 2007

[5] Brunton, S.L.; Rowley, C. W.; Kulkarni, S.R.; Clarkson, C., "Maximum Power Point Tracking for photovoltaic Optimization Using Ripple-Based Extremum Seeking Control", Power Electronics , IEEE Transactions on, vol. 25, pp.2531-2540, Oct. 2010

[6] Bazzi, A.M., and Krein, P. T., , "Concerning "Maximum Power Point Tracking fot photovoltaic Optimization Using Ripple-Based Extremum Seeking Control", Power Electronics , IEEE Transactions on, vol. 26, pp.1611-1612, Jun. 2011

[7] Leyva, R.; Olalla Martinez, C.; Zazo, H.; Cabal, C.; Cid Pastor, A; Queinnec, I.; Alonso, C; "MPPT based on Sinusoidal Extremum Seeking Control in PV Generation", International Journal of Photoenergy, in Press.

[8] Moase, W.H.; Manzie, C.; Brear, M.J. , "Newton-Like Extremum-Seeking for the Control of Thermoacoustic Instability", Automatic Control, IEEE Transactions on, vol. 55, pp.2094-2105, Sept. 2010

[9] Leyva, R.; Artillan, Ph.; Cabal, C.; Estibals, B.; Alonso, C. "Dynamic Performance of MPPT Circuits using Sinusoidal Extremum Seeking Control for PV Generation", Internacional Journal of Electronics, Vol. 94, pp. 529-542, Apr. 2011

\section{Conclusions}

The article reviews the technique named Newton-like Extremum Seeking Control and adapts it as MPPT circuit for photovoltaic systems. This technique uses the Hessian or curvature of the panel power to seek the maximum point. We describe the functional blocks of the proposed MPPT. Also, the paper compares this MPPT approach with an approach based on common Extremum Seeking 